\title{
Article
}

\section{Computing multiplicative topological indices of some chemical nenotubes and networks}

\author{
Zaryab Hussain ${ }^{1,2, *}$, Ahsan $^{3}$ and Shahid Hussain Arshad ${ }^{4}$ \\ 1 Department of Mathematics, Punjab College of Commerce New Campus Faisalabad Pakistan. \\ 2 Department of Mathematics, Government College University Faisalabad Pakistan. \\ 3 Superior Group of Colleges Faisalabad Campus, Faisalabad Pakistan.; ahsanulhaq8006@gmail.com \\ 4 Department of Applied Sciences, National Textile University Faisalabad Pakistan.; shahidarshad@ntu.edu.pk \\ * Correspondence: zaryabhussain2139@gmail.com; Tel.: +923207488346
}

Received: 25 July 2019; Accepted: 22 August 2019; Published: 6 October 2019

\begin{abstract}
The aim of this paper is to calculate the multiplicative topological indices of Zigzag polyhex nanotubes, Armchair polyhex nanotubes, Carbon nanocone networks, two dimensional Silicate network, Chain silicate network, six dimensional Hexagonal network, five dimensional Oxide network and four dimensional Honeycomb network.
\end{abstract}

Keywords: Chemical graph theory, multiplicative topological index, degree.

MSC: 05C05, 05C12, 05C50.

\section{Introduction}

$\mathbf{N}$ owadays graph theory is one of the most ironic and cited branch of mathematics due to its direct applications in our daily life. It is widely used in Computer networking and Chemistry. The area of graph theory related to Chemistry known as Chemical graph theory. This term firstly introduced by Balaban in book [1] in 1976. After it in 1991, Bonchev discussed more concepts in book [2] and in the book [3] by Trinajstić, we found a facet ideas about chemical graph theory its uses and applications in our daily life.

In the recent few years, lot of work has been done in chemical graph theory like in [4], Ali et al. calculated the topological indices of some chemical compounds. Pattabiraman and Suganya in [5] and Kanabur in [6] calculated topological indices of some well known graphs. The concept of multiplicative topological indices of graphs was given in [7-10]. In [11], Kahasy et al. calculate atom bond connectivity temperature index of some important organic compounds. Topological indices of some families of nanostar have been calculated in [12]. He and Jiang, in [13] calculated degree resistance distance of some trees. Degree-based multiplicative Atom-bond Connectivity index of some Nanostructures has been discussed [14]. In 2018, Hussain and Sabar [15] calculated multiplicative topological indices of single-walled titania nanotube. In [16], Kulli calculated some topological indices of two dimensional Silicate network, Chain silicate network, six dimensional Hexagonal network, five dimensional Oxide network and four dimensional Honeycomb network. Recently, Kulli [17] computed some topological indices of Zigzag polyhex nanotubes, Armchair polyhex nanotubes and Carbon nanocone networks. The main motivation of this work directly came from the papers [15-17].

\section{Preliminaries}

Let $G(V(G), E(G))$ be a finite, simple and connected graph with $V(G)=\left\{v_{1}, v_{2}, \ldots, v_{n}\right\}$ is the set of vertices and $E(G)=\left\{e_{1}, e_{2}, \ldots, e_{m}\right\}$ is the set of edges among the vertices of the graph. Consider a geodesic metric $d_{G}: V(G) \times V(G) \rightarrow \mathbb{R}$ defined as $d_{G}(u, v)$ is the number of edges between $u$ and $v$ in shortest path for any $u, v \in V(G)$. All the vertices which are exactly at distance 1 from $u \in V(G)$ are neighborhoods of $u$ in graph $G$ and collection of that vertices is called neighborhood set of $u$ in graph $G$ written as $N_{G}(u)$. Cardinality of neighborhood set of $u \in V(G)$ is called degree of $u$ in graph $G$ and in this paper it is denoted as $\xi_{u}$. 
First multiplicative Zagreb index is defined as:

$$
I I_{1}^{*}(G)=\prod_{r t \in E(G)}\left(\xi_{r}+\xi_{t}\right) .
$$

Second multiplicative Zagreb index is defined as:

$$
I I_{2}(G)=\prod_{r t \in E(G)}\left(\xi_{r} \cdot \xi_{t}\right) .
$$

The multiplicative first and second hyper-Zagreb indices are defined as:

$$
\begin{aligned}
& H I_{1}(G)=\prod_{r t \in E(G)}\left(\xi_{r}+\xi_{t}\right)^{2} . \\
& H I_{2}(G)=\prod_{r t \in E(G)}\left(\xi_{r} \cdot \xi_{t}\right)^{2} .
\end{aligned}
$$

First and second multiplicative generalized Zagreb indices are the generalized form of first and second multiplicative Zagreb indices as well as first and second multiplicative hyper-Zagreb indices. First and second multiplicative generalized Zagreb indices are defined as:

$$
\begin{aligned}
& M Z_{1}^{\alpha}(G)=\prod_{r t \in E(G)}\left(\xi_{r}+\xi_{t}\right)^{\alpha} . \\
& M Z_{2}^{\alpha}(G)=\prod_{r t \in E(G)}\left(\xi_{r} \cdot \xi_{t}\right)^{\alpha} .
\end{aligned}
$$

Multiplicative sum and product connectivity indices are defined as:

$$
\begin{aligned}
& \operatorname{SCII}(G)=\prod_{r t \in E(G)} \frac{1}{\sqrt{\xi_{r}+\xi_{t}}} . \\
& \operatorname{PCII}(G)=\prod_{r t \in E(G)} \frac{1}{\sqrt{\xi_{r} \cdot \xi_{t}}} .
\end{aligned}
$$

The multiplicative atomic bond connectivity index and geometric arithmetic index are defined as:

$$
\begin{aligned}
& A B C I I(G)=\prod_{r t \in E(G)} \sqrt{\frac{\xi_{r}+\xi_{t}-2}{\xi_{r} \cdot \xi_{t}} .} \\
& G^{*} A I I(G)=\prod_{r t \in E(G)}\left(\frac{2 \sqrt{\xi_{r} \cdot \xi_{t}}}{\xi_{r}+\xi_{t}}\right) .
\end{aligned}
$$

The general multiplicative geometric arithmetic index is defined as:

$$
G^{*} A^{\alpha} I I(G)=\prod_{r t \in E(G)}\left(\frac{2 \sqrt{\xi_{r} \cdot \xi_{t}}}{\xi_{r}+\xi_{t}}\right)^{\alpha} .
$$

Fact 1. Let $\eta_{1}, \eta_{2}, \ldots, \eta_{n}$ be a sequence. Then

$$
\prod_{i=1}^{n}\left(\eta_{i}\right)^{\gamma}=\left(\prod_{i=1}^{n} \eta_{i}\right)^{\gamma},
$$

where $\gamma$ is a constant. 
Proposition 2. Let $G(V(G), E(G))$ be any simple, connected and finite graph. Then

$$
\operatorname{SCII}(G)=\left(I I_{1}^{*}(G)\right)^{-\frac{1}{2}} .
$$

\section{Proof.}

$$
\begin{aligned}
\operatorname{SCII}(G) & =\prod_{r t \in E(G)} \frac{1}{\sqrt{\xi_{r}+\xi_{t}}} \\
& =\left(\frac{1}{\sqrt{\xi_{r}+\xi_{t}}}\right)^{|E(G)|} \\
& =\left(\xi_{r}+\xi_{t}\right)^{-\frac{E(G) \mid}{2}} \\
& =\left(\prod_{r t \in E(G)}\left(\xi_{r}+\xi_{t}\right)\right)^{-\frac{1}{2}} \\
& =\left(I I_{1}^{*}(G)\right)^{-\frac{1}{2}} .
\end{aligned}
$$

Proposition 3. Let $G(V(G), E(G))$ be any simple, connected and finite graph. Then

$$
\operatorname{PCII}(G)=\left(I I_{2}(G)\right)^{-\frac{1}{2}} .
$$

\section{Proof.}

$$
\begin{aligned}
\operatorname{PCII}(G) & =\prod_{r t \in E(G)} \frac{1}{\sqrt{\xi_{r} \cdot \xi_{t}}} \\
& =\left(\frac{1}{\sqrt{\xi_{r} \cdot \xi_{t}}}\right)^{|E(G)|} \\
& =\left(\xi_{r} \cdot \xi_{t}\right)^{-\frac{|E(G)|}{2}} \\
& =\left(\prod_{r t \in E(G)}\left(\xi_{r} \cdot \xi_{t}\right)\right)^{-\frac{1}{2}} \\
& =\left(I_{2}(G)\right)^{-\frac{1}{2}} .
\end{aligned}
$$

For detail concepts of topological indices on graphs we refer $[10,15]$.

\section{Main results}

\subsection{Zigzag Polyhex Nanotubes}

Zigzag polyhex nantube is denoted as $\operatorname{TUZC}_{6}[p, q]$, where $p$ is the number of hexagons in a row and $q$ is the number of hexagons in a column. A 2-Dimensional networks of $\operatorname{TUZC}_{6}[p, q]$ is shown in Figure 1. Let $G(V(G), E(G))$ be the graph of a $(p, q)$ dimensional zigzag polyhex nantube. It is easy to check that $|V(G)|=2 p(q+1)$ and $|E(G)|=p(3 q+2)$. In this structure there are two types of edges on the basis of their degrees, so we can decompose the the set of edges as $E(G)=E_{1}(G) \cup E_{2}(G)$, where

$$
\begin{aligned}
& E_{1}(G)=\left\{e=r t \in E(G) \mid \xi_{r}=2, \xi_{t}=3\right\}, \\
& E_{2}(G)=\left\{e=r t \in E(G) \mid \xi_{r}=3, \xi_{t}=3\right\} .
\end{aligned}
$$

It is easy to check that $\left|E_{1}(G)\right|=4 p$ and $\left|E_{2}(G)\right|=p(3 q-2)$. 


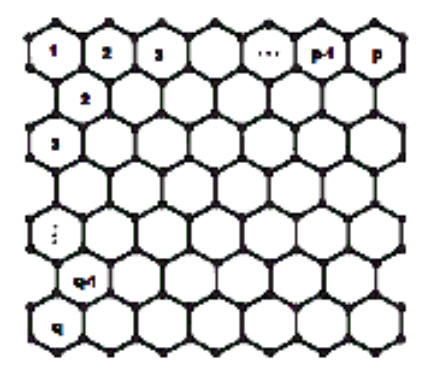

Figure 1. 2-Dimensional networks of $\operatorname{TUZC}_{6}[p, q]$

Theorem 4. Let $G(V(G), E(G))$ be the graph of Zigzag polyhex nanotube TUZC $6[p, q]$. Then the first multiplicative Zagreb index for $G$ is $2^{p(3 q-2)} \times 3^{p(3 q-2)} \times 5^{4 p}$.

Proof. The first multiplicative Zagreb index is:

$$
\begin{aligned}
I I_{1}^{*}(G) & =\prod_{r t \in E(G)}\left(\xi_{r}+\xi_{t}\right) \\
& =\prod_{r t \in E_{1}(G)}\left(\xi_{r}+\xi_{t}\right) \times \prod_{r t \in E_{2}(G)}\left(\xi_{r}+\xi_{t}\right) \\
& =(2+3)^{\left|E_{1}(G)\right|} \times(3+3)^{\left|E_{2}(G)\right|} \\
& =5^{4 p} \times 2^{3 p q-2 p} \times 3^{3 p q-2 p} \\
& =2^{p(3 q-2)} \times 3^{p(3 q-2)} \times 5^{4 p} .
\end{aligned}
$$

Theorem 5. Let $G(V(G), E(G))$ be the graph of Zigzag polyhex nanotube $\operatorname{TUZC}_{6}[p, q]$. Then the second multiplicative Zagreb index for $G$ is $2^{4 p} \times 3^{6 p q}$.

Proof. The second multiplicative Zagreb index is:

$$
\begin{aligned}
I I_{2}(G) & =\prod_{r t \in E(G)}\left(\xi_{r} \cdot \xi_{t}\right) \\
& =\prod_{r t \in E_{1}(G)}\left(\xi_{r} \cdot \xi_{t}\right) \times \prod_{r t \in E_{2}(G)}\left(\xi_{r} \cdot \xi_{t}\right) \\
& =(2 \cdot 3)^{\left|E_{1}(G)\right|} \times(3 \cdot 3)^{\left|E_{2}(G)\right|} \\
& =2^{4 p} \times 3^{4 p} \times 3^{6 p q-4 p} \\
& =2^{4 p} \times 3^{6 p q}
\end{aligned}
$$

Theorem 6. Let $G(V(G), E(G))$ be the graph of Zigzag polyhex nanotube $\operatorname{TUZC}_{6}[p, q]$. Then the multiplicative atomic bond connectivity index for $G$ is $2^{p(3 q-4)} \times 3^{p(2-3 q)}$.

Proof. The multiplicative atomic bond connectivity index is:

$$
\begin{aligned}
A B C I I(G) & =\prod_{r t \in E(G)} \sqrt{\frac{\xi_{r}+\xi_{t}-2}{\xi_{r} \cdot \xi_{t}}} \\
& =\prod_{r t \in E_{1}(G)} \sqrt{\frac{\xi_{r}+\xi_{t}-2}{\xi_{r} \cdot \xi_{t}}} \times \prod_{r t \in E_{2}(G)} \sqrt{\frac{\xi_{r}+\xi_{t}-2}{\xi_{r} \cdot \xi_{t}}}
\end{aligned}
$$




$$
\begin{aligned}
& =\left(\frac{2+3-2}{2.3}\right)^{\frac{\left|E_{1}(G)\right|}{2}} \times\left(\frac{3+3-2}{3.3}\right)^{\frac{\left|E_{2}(G)\right|}{2}} \\
& =\left(\frac{1}{2}\right)^{2 p} \times\left(\frac{2}{3}\right)^{p(3 q-2)} \\
& =2^{p(3 q-4)} \times 3^{p(2-3 q)} .
\end{aligned}
$$

Theorem 7. Let $G(V(G), E(G))$ be the graph of Zigzag polyhex nanotube $\operatorname{TUZC}_{6}[p, q]$. Then the multiplicative geometric arithmetic index for $G$ is $2^{6 p} \times 3^{2 p} \times 5^{-4 p}$.

Proof. The multiplicative geometric arithmetic index is:

$$
\begin{aligned}
G^{*} A I I(G) & =\prod_{r t \in E(G)}\left(\frac{2 \sqrt{\xi_{r} \cdot \xi_{t}}}{\xi_{r}+\xi_{t}}\right) \\
& =\prod_{r t \in E_{1}(G)}\left(\frac{2 \sqrt{\xi_{r} \cdot \xi_{t}}}{\xi_{r}+\xi_{t}}\right) \times \prod_{r t \in E_{2}(G)}\left(\frac{2 \sqrt{\xi_{r} \cdot \xi_{t}}}{\xi_{r}+\xi_{t}}\right) \\
& =\left(\frac{2 \sqrt{2 \cdot 3}}{2+3}\right)^{\left|E_{1}(G)\right|} \times\left(\frac{2 \sqrt{3.3}}{3+3}\right)^{\left|E_{2}(G)\right|} \\
& =\left(\frac{2.2^{\frac{1}{2}} \cdot 3^{\frac{1}{2}}}{5}\right)^{4 p} \times\left(\frac{2.3}{3+3}\right)^{2 p q-2 p} \\
& =2^{6 p} \times 3^{2 p} \times 5^{-4 p} .
\end{aligned}
$$

\subsection{Armchair Polyhex Nanotubes}

Carbon polyhex nantubes are those nantubes in which the cylindrical surface is entirely made up of hexagons. These type of carbon nantubes have very interesting thermal, electrical and mechanical properties, actually these are very stabile in nature. A 2 -dimensional networks of $T U A C_{6}[p, q]$ is shown in Figure 2. Let

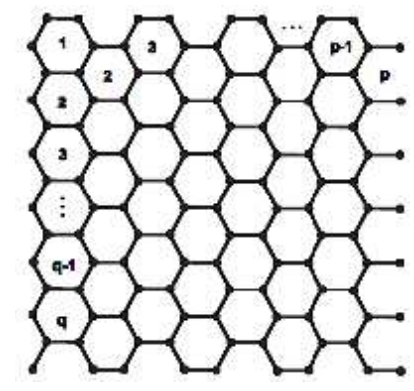

Figure 2. 2-dimensional networks of $\operatorname{TUAC}_{6}[p, q]$

$G(V(G), E(G))$ be the graph of Armchair polyhex nanotubes. It is easy to check that $|V(G)|=2 p(q+1)$ and $|E(G)|=p(3 q+2)$. In this structure there are three types of edges on the basis of their degrees, so we can decompose the the set of edges as $E(G)=E_{1}(G) \cup E_{2}(G) \cup E_{3}(G)$, where

$$
\begin{aligned}
& E_{1}(G)=\left\{e=r t \in E(G) \mid \xi_{r}=2, \xi_{t}=2\right\}, \\
& E_{2}(G)=\left\{e=r t \in E(G) \mid \xi_{r}=2, \xi_{t}=3\right\}, \\
& E_{3}(G)=\left\{e=r t \in E(G) \mid \xi_{r}=3, \xi_{t}=3\right\} .
\end{aligned}
$$


It is easy to check that $\left|E_{1}(G)\right|=p,\left|E_{2}(G)\right|=2 p$ and $\left|E_{3}(G)\right|=p(3 q-1)$. From this edge parttion, we can easily obtain the following results.

Theorem 8. Let $G(V(G), E(G))$ be the graph of Armchair polyhex nanotube $\operatorname{TUAC}_{6}[p, q]$. Then the first multiplicative Zagreb index for $G$ is $2^{p(1+3 q)} \times 3^{p(3 q-1)} \times 5^{2 p}$.

Theorem 9. Let $G(V(G), E(G))$ be the graph of Armchair polyhex nanotube $T_{U A C}[p, q]$. Then the second multiplicative Zagreb index for $G$ is $2^{4 p} \times 3^{6 p q}$.

Theorem 10. Let $G(V(G), E(G))$ be the graph of Armchair polyhex nanotube $T U A C_{6}[p, q]$. Then the multiplicative atomic bond connectivity index for $G$ is $\sqrt{2^{p(6 q-5)}} \times 3^{p(1-3 q)}$.

Theorem 11. Let $G(V(G), E(G))$ be the graph of Armchair polyhex nanotube $T_{U A C}[p, q]$. Then the multiplicative geometric arithmetic index for $G$ is $2^{3 p} \times 3^{p} \times 5^{-2 p}$.

\subsection{Carbon Nanocone Networks}

An $n$-dimensional one-pentagone nanocone is denoted as $\mathrm{CNC}_{5}[n]$, where $n$ is the number of hexagons layers encompassing the conical surface of nanocone and 5 denotes that there is a pentagon on the tip called its core. A 6 -dimensional one-pentagonal nanocone network is shown in the Figure 3. Now, $|V(G)|=$

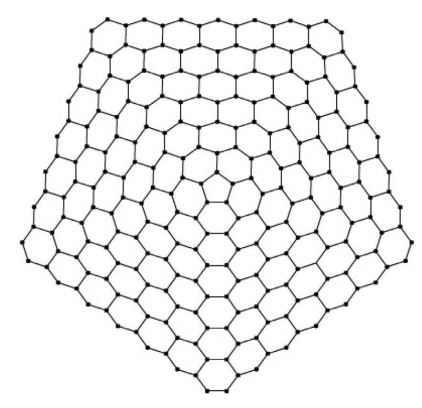

Figure 3. 6-dimensional one-pentagonal nanocone network

$5(n+1)^{2}$ and $|E(G)|=5\left(\frac{3}{2} n^{2}+\frac{5}{2} n+1\right)$. In this structure there are following three types of edges: $E(G)=$ $E_{1}(G) \cup E_{2}(G) \cup E_{3}(G)$, where

$$
\begin{aligned}
& E_{1}(G)=\left\{e=r t \in E(G) \mid \xi_{r}=2, \xi_{t}=2\right\}, \\
& E_{2}(G)=\left\{e=r t \in E(G) \mid \xi_{r}=2, \xi_{t}=3\right\}, \\
& E_{3}(G)=\left\{e=r t \in E(G) \mid \xi_{r}=3, \xi_{t}=3\right\} .
\end{aligned}
$$

It is easy to check that $\left|E_{1}(G)\right|=5,\left|E_{2}(G)\right|=10 n$ and $\left|E_{3}(G)\right|=5\left(\frac{3}{2} n^{2}+\frac{1}{2} n\right)$ and following results can be obtained immediately.

Theorem 12. Let $G(V(G), E(G))$ be the graph of Carbon nanocone networks $C N C_{5}[n]$. Then the first multiplicative Zagreb index for $G$ is $\sqrt{2^{15 n^{2}+5 n+20}} \times \sqrt{3^{15 n^{2}+5 n}} \times 5^{10 n}$.

Theorem 13. Let $G(V(G), E(G))$ be the graph of be the graph of Carbon nanocone networks $C_{N} C_{5}[n]$. Then the second multiplicative Zagreb index for $G$ is $2^{10(n+1)} \times 3^{15 n(n+1)}$.

Theorem 14. Let $G(V(G), E(G))$ be the graph of Carbon nanocone networks $C_{N} C_{5}[n]$. Then the multiplicative atomic bond connectivity index for $G$ is $\sqrt{2^{5\left(3 n^{2}-n-1\right)}} \times \sqrt{3^{-5 n(3 n+1)}}$.

Theorem 15. Let $G(V(G), E(G))$ be the graph of Carbon nanocone networks $C_{N} C_{5}[n]$. Then the multiplicative geometric arithmetic index for $G$ is $2^{15 n} \times 3^{5 n} \times 5^{-10 n}$. 


\subsection{Silicate Networks}

Silicates are formed by mixing of metal carbonates or metal oxides with sand. Silicate network is denoted as $S L_{n}$, where $n$ is the number of hexagons between the center and boundary of $S L_{n}$. A 2-dimensional

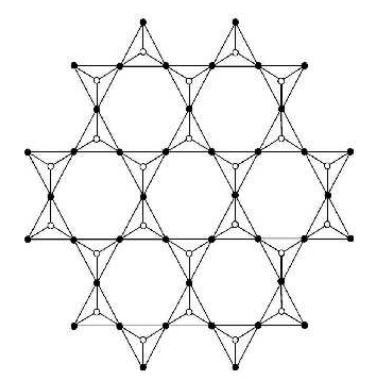

Figure 4. 2-dimensional silicate network

silicate network is shown in Figure 4. Let $G(V(G), E(G))$ be the graph of Silicate networks, then $|V(G)|=$ $3 n(5 n+1)$ and $|E(G)|=36 n^{2}$. In this structure there are three types of edges on the basis of their degrees, so we can decompose the the set of edges as $E(G)=E_{1}(G) \cup E_{2}(G) \cup E_{3}(G)$, where

$$
\begin{aligned}
& E_{1}(G)=\left\{e=r t \in E(G) \mid \xi_{r}=3, \xi_{t}=3\right\}, \\
& E_{2}(G)=\left\{e=r t \in E(G) \mid \xi_{r}=3, \xi_{t}=6\right\}, \\
& E_{3}(G)=\left\{e=r t \in E(G) \mid \xi_{r}=6, \xi_{t}=6\right\} .
\end{aligned}
$$

Now, $\left|E_{1}(G)\right|=6 n,\left|E_{2}(G)\right|=6 n(3 n+1)$ and $\left|E_{3}(G)\right|=6 n(3 n-2)$, so we have following results.

Theorem 16. Let $G(V(G), E(G))$ be the graph of Silicate network $S L_{n}$. Then the first multiplicative Zagreb index for $G$ is $2^{18 n(2 n-1)} \times 3^{6 n(9 n+1)}$.

Theorem 17. Let $G(V(G), E(G))$ be the graph of Silicate network $S L_{n}$. Then the second multiplicative Zagreb index for $G$ is $2^{18 n(3 n-1)} \times 3^{72 n^{2}}$.

Theorem 18. Let $G(V(G), E(G))$ be the graph of Silicate network $S L_{n}$. Then the multiplicative atomic bond connectivity index for $G$ is $2^{-9 n(2 n-1)} \times 3^{-36 n^{2}} \times 5^{3 n(3 n-2)} \times 7^{3 n(3 n+1)}$.

Theorem 19. Let $G(V(G), E(G))$ be the graph of Silicate network $S L_{n}$. Then the multiplicative geometric arithmetic index for $G$ is $2^{9 n(3 n+1)} \times 3^{-6 n(3 n+1)}$.

\subsection{Chain Silicate Networks}

Chain is obtained by arranging $n$ tetrahedral linearly. Chain silicate networks are denoted as $C S_{n}$.

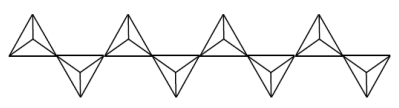

Figure 5. Chain silicate network

A chain silicate network is shown in Figure 5. Let $G(V(G), E(G))$ be the graph of chain Silicate networks, 
then $|V(G)|=3 n+1$ and $|E(G)|=6 n$. In this structure there are three types of edges on the basis of their degrees, so we can decompose the the set of edges as $E(G)=E_{1}(G) \cup E_{2}(G) \cup E_{3}(G)$, where

$$
\begin{aligned}
& E_{1}(G)=\left\{e=r t \in E(G) \mid \xi_{r}=3, \xi_{t}=3\right\}, \\
& E_{2}(G)=\left\{e=r t \in E(G) \mid \xi_{r}=3, \xi_{t}=6\right\}, \\
& E_{3}(G)=\left\{e=r t \in E(G) \mid \xi_{r}=6, \xi_{t}=6\right\} .
\end{aligned}
$$

It is easy to check that $\left|E_{1}(G)\right|=n+4,\left|E_{2}(G)\right|=2(2 n-1)$ and $\left|E_{3}(G)\right|=n-2$.

Theorem 20. Let $G(V(G), E(G))$ be the graph of Chain Silicate networks $C S_{n}$. Then the first multiplicative Zagreb index for $G$ is $2^{3 n} \times 3^{2(5 n-1)}$.

Theorem 21. Let $G(V(G), E(G))$ be the graph of Chain Silicate networks $C S_{n}$. Then the second multiplicative Zagreb index for $G$ is $2^{6(n-1)} \times 3^{12 n}$.

Theorem 22. Let $G(V(G), E(G))$ be the graph of Chain Silicate networks $C S_{n}$. Then the multiplicative atomic bond connectivity index for $G$ is $\sqrt{2^{-3 n+12}} \times \sqrt{3^{-12 n}} \times \sqrt{5^{n-2}} \times 7^{2 n-1}$.

Theorem 23. Let $G(V(G), E(G))$ be the graph of Chain Silicate networks $C S_{n}$. Then the multiplicative geometric arithmetic index for $G$ is $2^{3(2 n-1)} \times 3^{-2(2 n-1)}$.

\subsection{Hexagonal Networks}

It is known that there exist three regular plane tailings with composition of same kind of regular polygons such as triangles, squares and hexagonal. Triangular tiling is used in the construction of hexagonal networks. hexagonal network is denoted as $H X_{n}$, where $n$ is the number of vertices of in each side of hexagon.

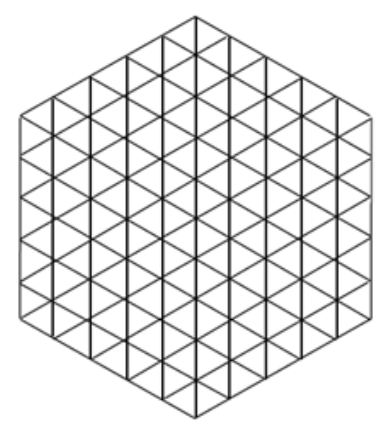

Figure 6. 6-diminsional hexagonal network

A 6-dimensional hexagonal network is shown in Figure 6. Let $G(V(G), E(G))$ be the graph of hexagonal network, then $|V(G)|=3 n^{2}-3 n+1$ and $|E(G)|=3\left(3 n^{2}-5 n+2\right)$. In this structure there are five type of edges on the basis of their degrees, so we can decompose the the set of edges as $E(G)=$ $E_{1}(G) \cup E_{2}(G) \cup E_{3}(G) \cup E_{4}(G) \cup E_{5}(G)$, where

$$
\begin{aligned}
& E_{1}(G)=\left\{e=r t \in E(G) \mid \xi_{r}=3, \xi_{t}=4\right\}, \\
& E_{2}(G)=\left\{e=r t \in E(G) \mid \xi_{r}=3, \xi_{t}=6\right\}, \\
& E_{3}(G)=\left\{e=r t \in E(G) \mid \xi_{r}=4, \xi_{t}=4\right\}, \\
& E_{4}(G)=\left\{e=r t \in E(G) \mid \xi_{r}=4, \xi_{t}=6\right\}, \\
& E_{5}(G)=\left\{e=r t \in E(G) \mid \xi_{r}=6, \xi_{t}=6\right\} .
\end{aligned}
$$

It is easy to check that $\left|E_{1}(G)\right|=12,\left|E_{2}(G)\right|=6,\left|E_{3}(G)\right|=6(n-3),\left|E_{4}(G)\right|=12(n-2)$ and $\left|E_{5}(G)\right|=$ $3\left(3 n^{2}-11 n+10\right)$. 
Theorem 24. Let $G(V(G), E(G))$ be the graph of Hexagonal Network $H X_{n}$. Then the first multiplicative Zagreb index for $G$ is $2^{18\left(n^{2}-2 n-1\right)} \times 3^{3\left(3 n^{2}-11 n+14\right)} \times 5^{12(n-2)} \times 7^{12}$.

Theorem 25. Let $G(V(G), E(G))$ be the graph of Hexagonal Network $H X_{n}$. Then the second multiplicative Zagreb index for $G$ is $2^{6\left(3 n^{2}-n-9\right)} \times 3^{6\left(3 n^{2}-9 n+10\right)}$.

Theorem 26. Let $G(V(G), E(G))$ be the graph of Hexagonal Network $H X_{n}$. Then the multiplicative atomic bond connectivity index for $G$ is $\sqrt{2^{-3\left(3 n^{2}-5 n+2\right)}} \times 3^{-3\left(3 n^{2}-10 n+13\right)} \times \sqrt{5^{3\left(3 n^{2}-11 n+14\right)}} \times 7^{3}$.

Theorem 27. Let $G(V(G), E(G))$ be the graph of Hexagonal Network $H X_{n}$. Then the multiplicative geometric arithmetic index for $G$ is $2^{3(6 n-1)} \times 3^{6(n-2)} \times 5^{-12(n-2)} \times 7^{-12}$.

\subsection{Oxide Networks}

An oxide network is denoted as $O X_{n}$, where $n$ is the number of dimensions. A 5-diminsional oxide

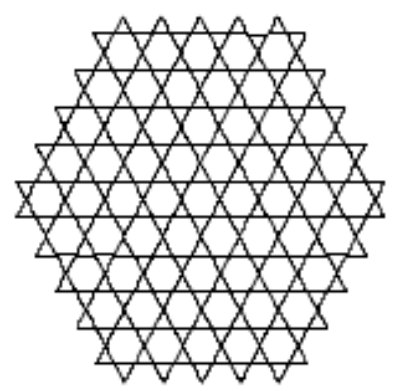

Figure 7. 5-diminsional oxide network

network is shown in Figure 7. Let $G(V(G), E(G))$ be the graph of oxide network, then $|V(G)|=9 n^{2}+3 n$ and $|E(G)|=18 n^{2}$. In this structure there are two type of edges on the basis of their degrees, so we can decompose the the set of edges such as $E(G)=E_{1}(G) \cup E_{2}(G)$, where

$$
\begin{aligned}
& E_{1}(G)=\left\{e=r t \in E(G) \mid \xi_{r}=2, \xi_{t}=4\right\}, \\
& E_{2}(G)=\left\{e=r t \in E(G) \mid \xi_{r}=4, \xi_{t}=4\right\} .
\end{aligned}
$$

It is easy to check that $\left|E_{1}(G)\right|=12 n$ and $\left|E_{2}(G)\right|=6 n(3 n-2)$.

Theorem 28. Let $G(V(G), E(G))$ be the graph of Oxide Network $O X_{n}$. Then the first multiplicative Zagreb index for $G$ is $2^{6 n(9 n-4)} \times 3^{12 n}$.

Theorem 29. Let $G(V(G), E(G))$ be the graph of Oxide Network $O X_{n}$. Then the second multiplicative Zagreb index for $G$ is $2^{12 n(6 n-1)}$.

Theorem 30. Let $G(V(G), E(G))$ be the graph of Oxide Network $O X_{n}$. Then the multiplicative atomic bond connectivity index for $G$ is $2^{-3 n(9 n-4)} \times 3^{3 n(3 n-2)}$.

Theorem 31. Let $G(V(G), E(G))$ be the graph of Oxide Network $O X_{n}$. Then the multiplicative geometric arithmetic index for $G$ is $2^{18 n} \times 3^{-12 n}$.

\subsection{Honeycomb Networks}

If we recursively use hexagonal tiling in a particular pattern, honeycomb networks are formed. A honeycomb is denoted as $H C_{n}$, where $n$ is the number of hexagons between the central and boundary hexagon. 


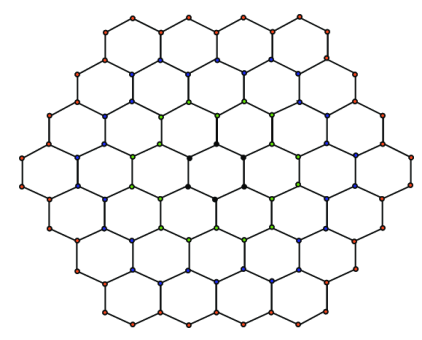

Figure 8. 4-diminsional honeycomb network

A 4-dimensional honeycomb network is shown in Figure 8. Let $G(V(G), E(G))$ be the graph of honeycomb network, then $|V(G)|=6 n^{2}$ and $|E(G)|=3 n(3 n-1)$. In this structure there are three type of edges on the basis of their degrees, so we can decompose the the set of edges as $E(G)=E_{1}(G) \cup E_{2}(G) \cup E_{3}(G)$, where

$$
\begin{aligned}
& E_{1}(G)=\left\{e=r t \in E(G) \mid \xi_{r}=2, \xi_{t}=2\right\}, \\
& E_{2}(G)=\left\{e=r t \in E(G) \mid \xi_{r}=2, \xi_{t}=3\right\}, \\
& E_{3}(G)=\left\{e=r t \in E(G) \mid \xi_{r}=3, \xi_{t}=3\right\} .
\end{aligned}
$$

It is easy to check that $\left|E_{1}(G)\right|=6,\left|E_{2}(G)\right|=12(n-1)$ and $\left|E_{3}(G)\right|=3\left(3 n^{2}-5 n+2\right)$.

Theorem 32. Let $G(V(G), E(G))$ be the graph of Honeycomb Network $H C_{n}$. Then the first multiplicative Zagreb index for $G$ is $2^{3\left(3 n^{2}-5 n+6\right)} \times 3^{3\left(3 n^{2}-5 n+2\right)} \times 5^{12(n-1)}$.

Theorem 33. Let $G(V(G), E(G))$ be the graph of Honeycomb Network $H C_{n}$. Then the second multiplicative Zagreb index for $G$ is $2^{12 n} \times 3^{18 n(n-1)}$.

Theorem 34. Let $G(V(G), E(G))$ be the graph of Honeycomb Network $H C_{n}$. Then the multiplicative atomic bond connectivity index for $G$ is $2^{3\left(3 n^{2}-7 n+3\right)} \times 3^{-3\left(3 n^{2}-5 n+2\right)}$.

Theorem 35. Let $G(V(G), E(G))$ be the graph of Honeycomb Network $H C_{n}$. Then the multiplicative geometric arithmetic index for $G$ is $2^{18(n-1)} \times 3^{6(n-1)} \times 5^{-12(n-1)}$.

Remark 1. We can compute the following easily by using Propositions 2 and 3.

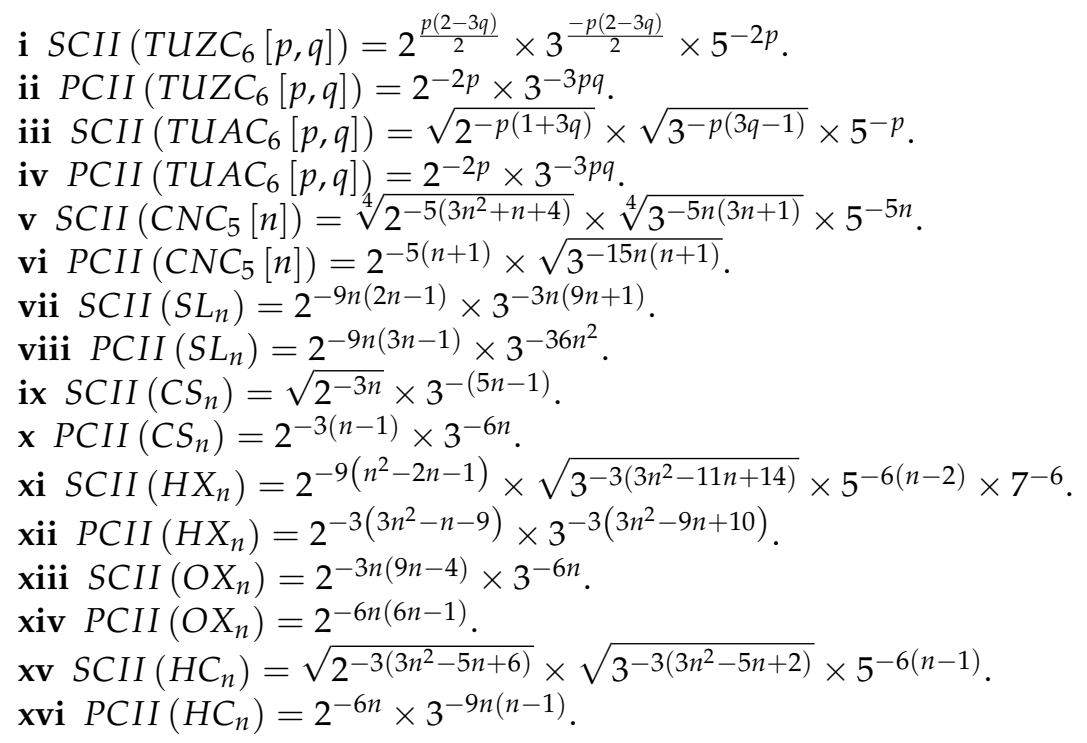

Remark 2. We can compute the following easily by using Fact 1. 


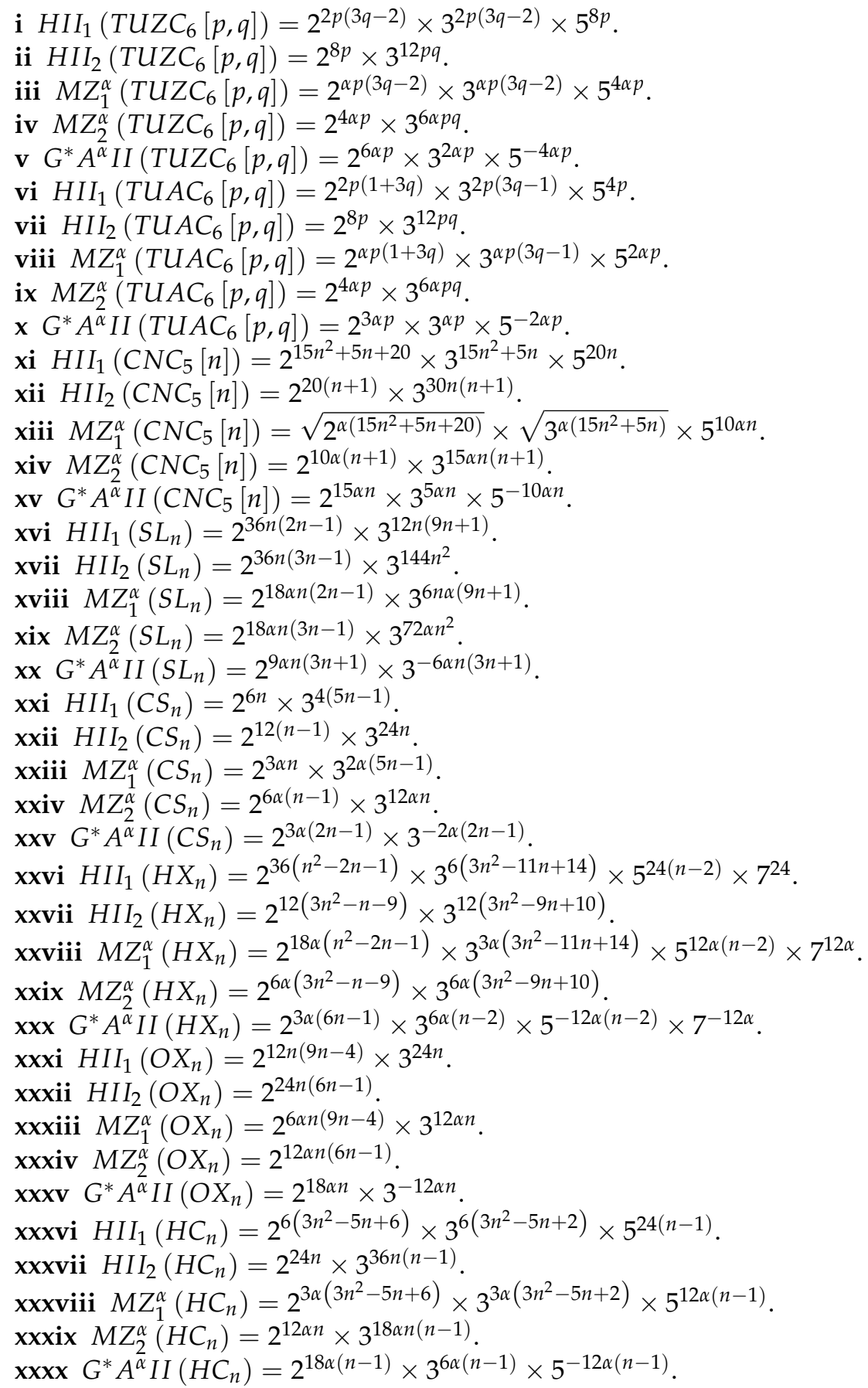

Acknowledgments: We greatly thank the referees for careful reading and helpful suggestions that led to many improvements.

Author Contributions: All authors contributed equally to the writing of this paper. All authors read and approved the final manuscript.

Conflicts of Interest: "The authors declare no conflict of interest."

\section{References}

[1] Balaban, A. T. (1985). Applications of graph theory in chemistry. Journal of chemical information and computer sciences, 25(3), 334-343.

[2] Bonchev, D. (1991). Chemical graph theory: introduction and fundamentals (Vol. 1). CRC Press.

[3] Trinajstić, N. (2018). Chemical graph theory. Routledge.

[4] Ali, H., Baig, A. Q., \& Shafiq, M. K. (2017). On topological properties of boron triangular sheet BTS $(m, n)$, borophene chain $B_{36}(n)$ and melem chain $M C(n)$ nanostructures. Journal of Mathematical Nanoscience, 7(1), 39-60. 
[5] Pattabiraman, K., \& Suganya, T. (2018). Edge version of some degree based topological descriptors of graphs. Journal of Mathematical Nanoscienese, 8(1), 1-12.

[6] Kanabur, R. (2018). On certain cegree-based topological indices of armchair poly-hex nanotubes. Journal of Mathematical Nanoscienese, 8(1), 19-25.

[7] Gutman, I. (2011). Multiplicative Zagreb indices of trees. Bulletin of Society of Mathematicians Banja Luka, 18, 17-23.

[8] Iranmanesh, A., Hosseinzadeh, M. A., \& Gutman, I. (2012). On multiplicative Zagreb indices of graphs. Iranian Journal of Mathematical Chemistry, 3(2), 145-154.

[9] Ghorbani, M., \& Azimi, N. (2012). Note on multiple Zagreb indices. Iranian Journal of Mathematical Chemistry, 3(2), 137-143.

[10] Hussain, Z., Ijaz, N., Tahir, W., Butt, M. T., \& Talib, S. (2018). Calculating Degree Based Multiplicative Topological indices of Alcohol. Asian Journal of Applied Science and Technology 4(2), 132-139.

[11] Kahasy, A.T., Narayankar, K., \& Selvan, D. (2018). Atom bond connectivity temperature index. Journal of Mathematical Nanoscienese, 8(2), 67-75.

[12] Siddiqui, M. K., Rehman, N. A., \& Imran, M. (2018). Topological indices of some families of nanostar dendrimers. Journal of Mathematical Nanoscienese, 8(2), 91-103.

[13] He, F. \& Jiang, X. (2018). Degree resistance distance of trees with given perameters. Transaction on Combinatorics, 7(4), $11-24$.

[14] Gao, W., Jamil, M. K., Nazeer, W., \& Amin, M. (2017). Degree-Based Multiplicative Atom-bond Connectivity Index of Nanostructures. International Journal of Applied Mathematics, 47(4).

[15] Hussain, Z., \& Sabar, S. (2018). On multiplicative degree based topological indices of single-walled titania nanotubes. Journal of Mathematical Nanoscienese, 8(1), 41-54.

[16] Kulli, V. R. (2017). Computation of some topological indices of certain networks. International Journal of Mathematical Archive, 8(2), 99-106.

[17] Kulli,V. R. (2019). F-Indices of chemical networks. International Journal of Mathematical Archivem, 10(3), 21-30.

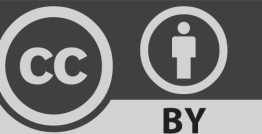

(C) 2019 by the authors; licensee PSRP, Lahore, Pakistan. This article is an open access article distributed under the terms and conditions of the Creative Commons Attribution (CC-BY) license (http://creativecommons.org/licenses/by/4.0/). 